\title{
Notes on Uses of the Bird Respirator Treatment by Physiotherapy
}

By J. MALCOLM, M.N.Z.S.P.

Patients who benefit from treatment with the Bird Respirator fall into three main groups.

1. The Co-operative patient.

2. The Unco-operative patient.

3. The Unconscious patient.

\section{Group 1.}

This group falls into two sections again.

(a) Chronic Lung Diseases: Bronchitis, Emphysema, Asthma.

(b) Post Operative Patient, and acute respiratory diseases.

(a) Chronic Lung Diseases.

Aims of Treatment.

(i) Increase the flow of air through congested bronchioles.

(ii) Fully expand stiff and rigid chest and increase the movement of a fixed or partially fixed diaphragm.

(iii) Introduce nebulized broncho-dilators into the bronchioles for relief of oedema and spasm.

(iv) Clear mucus and secretions from the chest.

Methods of Treatment.

(i) Position the patient where possible. Use postural drainage positions to aid the movement of mucus to the main bronchus where it can be easily coughed out.

(ii) Use the mouth piece of the Bird fitted to the nebulizer (into which the broncho-dilator prescribed, or distilled water, has been added).

(iii) Set dials of machine as follows:

Flowrate: Use a long flow rate depending on the patient's capacity (i.e. size of chest, amount of effective lung tissue left, age of patient). A long flow rate is between 7-15. This introduces positive pressure slowly so that air pushes gently past secretions to expand collapsed alveoli and enables patient to cough out moistened secretions lying in the airway. Even if the patient is too depressed to cough, gases flowing out of the lung gradually propel secretions out of the peripheral airways.

Respiratory Pressure: Set again according to patient's capacity: 15 is considered normal for an adult, but a man with healthy lungs will take up to 25 comfortably.
Air Mix: This should be pulled full out to give $40^{\circ} \mathrm{O}_{2}$ and $60^{\circ}$ atmospheric air.

Respiration Effort.

Set at 40 , unless patient very weak. This indicates the amount of effort the patient makes to trigger the respirator, and should be made fairly difficult for the voluntary patient.

(iv) Instruct patient in use: Hold the mouth piece in his mouth, gently pinch his nostrils together so air does not escape through nose. Teach him to relax on inspiration and let all the air flow into lungs and not cheeks. Tell him not to block the flow of air by closing his throat or blocking the mouth piece with his tongue.

(v) Expiration: Encourage to breathe out as far as possible. Help by pushing on lower ribs and pushing diaphragm up. (If patient is tipped the weight of the viscera will also help diaphragm to move upwards).

Footnote: With asthmatics, lower the inspiratory pressure; use prescribed bronchodilator and encourage long, relaxed expiration. If at first you wish to retard the expiratory phase to teach patient to expire slowly and in a relaxed fashion, use the "retard guard" which you will find attached to machine.

(b) Post Operative Patient and Acute Respiratory Diseases.

In this group is included-

1. Post operative laparotomy where there is chronic chest discase.

2. Thorocotomy and lobectomy.

3. Pneumothorax.

4. Haemothorax.

5. Spinal Injuries.

6. Injuries of Chest.

7. Atelectasis.

8. Acute infection of chronic chest conditions.

Aims of Treatment.

(i) Maintain adequate expansion of lungs and good interchange of air.

(ii) Increase flow of air into collapsed bronchioles. This is effected by the positive pressure on inspiration.

(iii) Assist expectoration. 
(iv) Decrease the amount of pain and effort necessary to keep lung expanded.

(v) Re-establish a good cough reflex and good voluntary breathing habits where possible.

Methods of Treatment.

(i) If possible treat the patient pre-operatively. Teach him how to use the Bird with the mouth piece. Show him the positions you will use and teach him to cough "with" the respirator (i.e. use the respirator for inspiration; remove the mouth piece, and cough).

(ii) Measure the tidal volume pre-operatively with a spirometer. This will give you some indication for setting the inspiratory pressure of the machine afterwards.

(iii) Position the patient: When treating pneumothorax, haemothorax; thorocotomy and acute infections, lie patient down on the opposite side with a pillow under his chest. This helps to limit the intake of air to the good side: eliminates the spinal scoliosis which has nearly always developed; and aids expiration (the weight of the viscera pushes up on the diaphragm).

Back cases cannot be turned but in all cases tip the bed.

(iv) Set the machine 1. Slow inspiratory time flow.

2. Effort: high.

3. Pressure according to patient and condition.

(v) If it does not cause too much pain in the first stage, assist expiration. Encourage patient to force expiration. Mucus and secretions should move towards the pharynx easily.

(vi) Use the nebulizer to soften and loosen secretions.

(vii) The patient should take over from the Bird as soon as the acute stage is over. Even if the collapsed lung is well expanded with the Bird, the patient may not be able to expand it well voluntarily. Teach this with the usual post-operative routine.

Group 2. The Unco-operative Patient.

This is the patient in acute respiratory failure or acidosis. $\mathrm{He}$ still has a cough reflex and a patent airway. Due to carbon-dioxide narcosis he may be dazed, belligerent or psychotic, cyanosed, gasping for breath or unwilling to co-operate.

Aims of Treatment.

(i) Restore adequate tidal air flow as quickly as possible.

(ii) Drainage of secretions.

(iii) Restore breathing to a point where patient can provide enough alveolar gas exchange unassisted and spontaneously.

Method.

(i) Try to stop patient's panic. Remain calm yourself.

(ii) Lie the patient supine with shoulders raised $20^{\circ}$ and head tilted backwards (this keeps the larynx open). Pull the patient's jaw forward.

(iii) An airway may be necessary.

(iv) Select a well fitting mask to cover nose and mouth. Hold this firmly so that no air escapes.

(v) Make sure the nebulizer is full.

(vi) Set the machine 1. Easy effort.

$$
\text { 2. Small pressure. }
$$

3. Short flow rate.

As the patient's condition improves, lower the shoulders and tip the end of the bed so secretions can drain more easily. Then gradually increase the pressure and flowrate and the inspiratory effort.

(vii) Aid expiration.

(viii) When the patient coughs, encourage him and sit him up.

(ix) The patient should stay on the respirator until his condition improves sufficiently to take over himself.

(x) The machine should be used intermittently, as for the co-operative patient, when he has recovered.

(xi) Teach breathing exercises as for the co-operative patient.
Tracheostomy Patient.

This is again divided into two groups.

(1) Flaccid, unconscious patient who is not breathing voluntarily. (i.e. Tetanus, when patient has been given large doses of muscle relaxant; over dose of drugs, polio; high spinal chord lesions affecting muscles of respiration). The patient usually has little or no cough reflex.

\section{Treatment.}

The patient will be on continuous artificial respiration and taken off only for suctioning and artificial coughing with ambru bag.

When furst applying machine to patient, set all controls at 15 (This now includes the automatic respiratory control knob as the patient is not triggering the machine) N.B. do not use the negative pressure switch.

When the machine is going and everything is set up, charge the settings as required and take a tidal volume reading with a spirometer.

Check several things half hourly.

1. Spirometer readings should remain the same, if they do not it indicates $(a)$ block in tubing,

(b) leak in circuit.

2. Check the cuff. It should allow a very small amount of air to leak past to propel secretions around it upwards (on inspiration) where they can be sucked from the pharynx.

3. Half hourly or hourly let the cuff, down quickly: suction: and blow cuff up again. Thishrests the mucus membrane around the cuff and allows secretions to move down into the trachea where they can be sucked out. The respirator will have to be disconnected while suctioning.

4. Check the nebulizer: it must never run dry.

5. Check the tubes of the respirator for water accumulation; shake them dry when necessary.

6. Always watch for respirator jumping-this indicates obstruction.

7. When suctioning introduce $15 \mathrm{cc}$ of sterile saline into the tracheostomy to soften mucus and prevent crusting.

8. If an $\mathrm{O}_{2}$ cylinder is being used, check for pressure. This should always remain higher than $64 \mathrm{lbs} . \quad(=$ to $500 \mathrm{cc} \mathrm{O}_{2}$ content) of pressure.

The Artificial Cough.

Twice daily, or more if necessary, transfer patient to the ambru bag. Tip the bed for a full tip. Two operators are necessary. One to squeeze the bag fully and slowly while the other presses sharply on the chest during the expiratory phase to effect a cough or sharp expiration. Suction during this when necessary.

(2) The Co-operative Tracheostomy Patient.

Treatment.

(i) While the patient is continually on the respirator encourage him to assist expiration and relax with the machine on inspiration.

(ii) Try to encourage the cough reflex while tipping and suctioning. If patient is very debilitated or the cough reflex is diminished it may be necessary to use the ambru bag and artificial cough.

(iii) Try to wean patient off machine as soon as his condition improves. Let the cuff down while patient is off machine and encourage breathing and coughing through the mouth as well as the tracheostomy. Then teach him to breathe through his mouth only by blocking off the tracheostomy with his finger. Start taking patient off machine for 5 minutes and if he shows no sign of distress gradually increase the time.

(iv) When patient is coughing well voluntarily and breathing through both tracheostomy and mouth the tube may be removed. Air will escape for a few days through incision. Teach breathing exercises and cough with pressure over incision site to prevent this.

(v) Throughout treatment continue to teach localized breathing exercises and postural drainage if necessary. 